\title{
A New Approach to the Construction of Dynamical Structure of our Galaxy
}

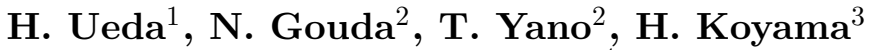 \\ and M. Sakagami ${ }^{4}$ \\ ${ }^{1}$ Faculty of Education and Human Studies, Akita University, Tegata-gakuen, Akita 010-0852, \\ Japan \\ email: ueda@ipc.akita-u.ac.jp \\ ${ }^{2}$ National Astronomical Observatory, Mitaka, Tokyo 181-8588, Japan \\ email:naoteru.gouda@nao.ac.jp,yano.t@nao.ac.jp \\ ${ }^{3}$ Graduate School of Human and Environmental Studies, Kyoto University, Kyoto 606-8501, \\ Japan \\ email: sakagami@grav.mbox.media.kyoto-u.ac.jp \\ ${ }^{4}$ Department of Physics, Waseda University, Shinjuku, Tokyo 169-8555, Japan \\ email: koyama@gravity.phys.waseda.ac.jp
}

Information about positions and velocities of stars that will be gained in the era of GAIA is crucial for determining dynamical structure in our Galaxy. The distribution function of all component objects in our Galaxy is fundamental for describing its dynamics. However, only the distribution function of observable stars is obtained from space astrometry observations, and it is therefore necessary to develop theoretical studies of how to construct the distribution function of all matter including dark matter and unobservable stars using astrometric data of observable stars. This procedure falls into three categories.

1) Torus Fitting: In the first step, we find action variables $\mathbf{J}$ in any gravitational potential (Hamiltonian) whose system is almost integrable and considered to be a representation of our Galaxy. Concretely, we find the coordinate transformation between action variables and the Cartesian coordinates $(\mathbf{x}, \mathbf{p})$ in a model of our Galaxy. Although the action-angle variables $(\mathbf{J}, \theta)$ provide the most compact representation of a regular orbit, it is impossible to get analytically the coordinate transformation $(\mathbf{J}, \theta) \Leftrightarrow(\mathbf{x}, \mathbf{p})$ in an arbitrary potential. We therefore propose a new approach that numerically obtains action variables $\mathbf{J}$ and coordinate transformations $(\mathbf{J}, \theta) \Leftrightarrow(\mathbf{x}, \mathbf{p})$. It is called a "Torus Fitting method" and is an alternative of the Torus Construction method proposed by Binney and his collaborators.

2) Determination of a theoretical distribution function by M2M: In the second place, we construct the distribution function $f_{\text {matter }}\left(J_{1}, J_{2}, J_{3}\right)$ of all matter in the system, using action variables from the first step. We do this using the made-to-measure algorithm for constructing an $N$-body realization of an equilibrium stellar system (please refer to Yano's presentation for details).

3) Determination of a model of our Galaxy from the distribution function of observed stars, $f_{\text {obs }}(\mathbf{x}, \mathbf{p})$ : Finally, we have to determine a model of the gravitational potential (Hamiltonian) of our Galaxy. Hence we need to construct the distribution function of all matter including dark matter and unobservable stars using astrometric data that include the error in distance measurements and selection biases. In this procedure a technique that utilizes Hermite polynomials is used.

From the above procedure we obtain the distribution function of all matter using space astrometry observations. 